\title{
Efeito de herbicidas e fungicidas nas curvas de progresso e quantificação de danos causados pela ferrugem asiática da soja
}

\author{
Effect of herbicides and fungicides on progress curves and damage quantification caused \\ by soybean rust
}

\author{
Guy Mitsuyuki Tsumanuma ${ }^{\text {I*IV }}$ Ana Raquel Soares ${ }^{\mathrm{II}}$ Antonio Luiz Fancelli ${ }^{\mathrm{II}}$ \\ Marco Antonio Tavares Rodrigues ${ }^{\mathrm{I}, \mathrm{IV}}$ Edson Begliominiv $^{\mathrm{IV}}$
}

\section{RESUMO}

O fungo Phakopsora pachyrhizi Sydow \& Sydow representa grande ameaça à sojicultura nas principais regiões produtoras do mundo, onde significativas perdas foram relatadas. Na quantificação de danos causados, muitas são as variáveis a serem estudadas, como área abaixo da curva de progresso da doença (AACPD), absorção de luz da área foliar sadia (HAA) e duração da área foliar sadia (HAD), além das curvas de progresso da doença. Neste trabalho, objetivou-se verificar a influência de diferentes herbicidas e fungicidas no progresso da doença, bem como suas implicações nas variáveis referentes à área foliar sadia e à produtividade da cultura. Os tratamentos testados em duas cultivares ('MG/BR46 Conquista' e 'BRS Valiosa RR') foram: 1. Testemunha; 2. Testemunha com herbicidas; 3. Herbicidas e pyraclostrobin (V4) + pyraclostrobin + epoxiconazole (R2 e R5.1); 4. Herbicidas e pyraclostrobin (V4) + epoxiconazole (R2 e R5.1); e 5. Herbicidas (V4) + pyraclostrobin + epoxiconazole (R2 e R5.1). Os herbicidas utilizados em 'MG/BR-46 Conquista' foram sethoxydim, bentazon e chlorimuron-ethyl, sendo utilizado glyphosate na 'BRS Valiosa RR'. O modelo logístico foi o que melhor se adequou à severidade média das avaliações e à curva de progresso da doença. Para o terço inferior, posição do dossel mais propícia à doença, o modelo logístico com taxa variável foi o que mais se ajustou aos dados. A pulverização de pyraclostrobin + epoxiconazole reduziu a taxa de desenvolvimento de $\boldsymbol{P}$. pachyrhizi em relação à epoxiconazole. A utilização da mistura influenciou as variáveis AACPD, HAA e HAD, sendo estas consideradas apropriadas para a quantificação dos danos provocados pela ferrugem asiática. $\mathrm{Na}$ 'MG/BR-46 Conquista', a ação dos herbicidas afetaram temporariamente a área foliar, atingindo indiretamente as variáveis $H A A$ e $H A D$.

Palavras-chave: Glycine $\boldsymbol{m a x}$ (L.) Merrill, AACPD, HAA, HAD, estrobilurina, triazol.

\section{ABSTRACT}

Phakopsora pachyrhizi Sydow \& Sydow is the worst threat for soybean crop in the most important growing regions around the world, where great losses were observed. Many variables are studied in the quantification of damage such as area under the disease progress curve (AUDPC), healthy leaf area absorption (HAA), healthy leaf area duration (HAD), in addition to disease progress curves. The present research aimed to evaluate the effect of several herbicides and fungicides on the progress of the disease and its implications for variables related to healthy leaf area and yield. The treatments were evaluated in two cultivars (MG/BR-46 Conquista e BRS Valiosa RR): 1. Control; 2. Control with herbicides; 3. Herbicides and pyraclostrobin (V4) + pyraclostrobin + epoxiconazole (R2 and R5.1), 4. Herbicides and pyraclostrobin (V4) + epoxiconazole (R2 and R5.1). 5. Herbicides (V4) + pyraclostrobin + epoxiconazole (R2 and R5.1). The herbicides used in MG/BR46 Conquista were sethoxydim, bentazon and chlorimuronethyl; in BRS Valiosa RR, glyphosate was used. The logistic was the model that best adapted to the mean severity ratings and the disease progress curve. At the bottom of canopy, position more favorable to this disease, the logistic model with variable rate was the model that better explain the data. The spraying of strobilurin's group associated with triazole (pyraclostrobin + epoxiconazole) reduced the rate of asian rust development, in relation to the group of ergosterol biosynthesis inhibitors fungicide (epoxiconazole). The pyraclostrobin + epoxiconazole influenced the variables $A U D P C, H A A$ and $H A D$, and these can be considered suitable to quantify damage caused by this disease. In MG/BR-46 Conquista, the herbicides action affects temporarily the leaf area, affecting indirectly the HAA and $H A D$ variables.

Key words: Glycine $\max (L$.$) Merrill, AUDPC, HAA, HAD,$ strobilurin, triazole.

IPrograma de Pós-graduação em Fitotecnia, Escola Superior de Agricultura "Luiz de Queiroz" (ESALQ), Universidade de São Paulo (USP), CP 09, 13418-900, Piracicaba, SP, Brasil. E-mail: tsumanuma@yahoo.com.br. *Autor para correspondência.

IIDepartamento de Fitopatologia, ESALQ, USP, Piracicaba, SP, Brasil.

IIIDepartamento de Produção Vegetal, Piracicaba, SP, Brasil.

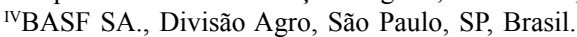




\section{INTRODUÇÃO}

O fungo Phakopsora pachyrhizi Syd. \& Syd., em razão de sua agressividade, causa perdas de produtividade da ordem de 10-90\% na Índia, $10-50 \%$ no sul da China, $23-90 \%$ em Taiwan e $40 \%$ no Japão (SINCLAIR \& HARTMAN, 1999). No Brasil, desde a safra 2000/2001, a incidência da "ferrugem asiática" tem causado perdas significativas na produtividade, contribuindo para o incremento dos custos de produção. Na safra de 2003/2004, a doença atingiu praticamente todas as áreas de plantio do país, exceto os Estados de Roraima e Amapá, gerando redução de 4,6 milhões de toneladas, o que corresponde ao valor de US\$1,22 bilhão de dólares (YORINORI \& LAZZAROTTO, 2004). O principal método de controle é realizado quimicamente, e no caso da ferrugem da soja, os fungicidas restringem-se aos grupos químicos: triazóis e estrobilurina, sendo recomendados antes do estabelecimento do patógeno.

A obtenção de estimativas confiáveis dos prejuízos causados pelos patógenos é considerada prérequisito essencial para o desenvolvimento de programas bem sucedidos de controle de doenças. Nesse sentido, a utilização de modelos matemáticos e de curvas de progresso da doença tem importância crescente, uma vez que permitem o monitoramento de doenças por meio de avaliações da severidade no tempo (BERGAMINFILHO, 1995).

As doenças foliares têm por característica diminuir a quantidade de tecido foliar sadio, ocasionando redução no rendimento, pela menor atividade fotossintética das folhas. Assim, modelos que substituem a intensidade de doença pela duração da área foliar sadia (HAD) ou absorção de luz da área foliar sadia (HAA) possibilitariam um melhor entendimento das relações entre doenças e seus danos na produtividade. Dessa forma, a produção de culturas doentes está diretamente relacionada com a área foliar sadia em determinado estádio de crescimento, com a duração da área foliar, ou com a quantidade acumulada de radiação interceptada pela área foliar sadia da planta. As variáveis HAA e HAD fornecem ainda parâmetros sobre o tamanho das plantas, a quantidade de luz absorvida e o efeito das doenças sobre as folhagens das plantas (GODOY, 1995).

Estudos acerca da relação entre AACPD, HAA e HAD com produtividade vêm sendo realizados. Segundo CALAÇA (2007), a variável que melhor se relaciona com o rendimento de soja é HAA, e tanto HAA, quanto HAD são variáveis melhores do que a severidade para serem usadas na quantificação de danos provocados pela ferrugem asiática na soja.
SCHMIDT et al. (2008), por sua vez, encontraram maior correlação entre produtividade e HAD do que com HAA. A utilização dessas variáveis está diretamente relacionada à produtividade porque consideram a área foliar fotossinteticamente ativa, ou seja, a área sadia que possibilita a absorção da radiação solar.

Recentemente, há relatos da ação indireta de herbicidas sobre a doença, mediante seus efeitos na morfologia e na fisiologia dos cultivos. Nesse sentido, deve-se relatar a existência de estudos sobre o controle das ferrugens da soja e do trigo, com uso de glyphosate (ANDERSON \& KOLMER, 2005; FENG et al., 2005), o qual teria efeito transitório na germinação dos esporos, provavelmente pela diluição desta nos tecidos em crescimento.

Ainda são incipientes os trabalhos que relacionam os efeitos conjuntos de herbicidas e fungicidas no controle da ferrugem asiática, razão pela qual se objetivou verificar a influência desses defensivos agrícolas, no progresso de P. pachyrhizi, bem como suas implicações nas variáveis referentes à área foliar sadia e produtividade de duas cultivares de soja.

\section{MATERIAL E MÉTODOS}

O experimento foi conduzido em condições de campo, irrigadas por aspersão, no município de Assaí, Estado do Paraná (latitude $23^{\circ} 34^{\prime} 50^{\prime \prime}$, longitude $50^{\circ} 81^{\prime} 00^{\prime \prime}$ e altitude de $590 \mathrm{~m}$ ), na safra 2006 . O solo da área experimental foi caracterizado como Nitossolo eutrófico típico, com relevo suave ondulado. As cultivares utilizadas foram: 'MG/BR 46 Conquista' (Convencional) e 'BRS Valiosa RR' (transgênica permite uso de glyphosate).

$\mathrm{O}$ delineamento experimental adotado foi blocos ao acaso, constituído de cinco tratamentos e quatro repetições, em duas cultivares de soja, as quais foram analisadas isoladamente. As parcelas experimentais foram constituídas de quatro fileiras de $10 \mathrm{~m}$ de comprimento, espaçadas de $0,45 \mathrm{~m}$, perfazendo área útil de $7,2 \mathrm{~m}^{2}$. Os tratamentos testados nas duas cultivares foram: 1 . Testemunha; 2 . Testemunha com herbicidas; 3 . Herbicidas e pyraclostrobin $\left(75 \mathrm{~g} \mathrm{ha}^{-1}\right)$ em V4 + pyraclostrobin + epoxiconazole $\left(66,5+25 \mathrm{~g}^{-} \mathrm{a}^{-}\right.$ 1) em R2 e R5.1; 4. Herbicida e pyraclostrobin $\left(75 \mathrm{~g} \mathrm{ha}^{-1}\right)$ em V4 + epoxiconazole (50g ha-1) em R2 e R5.1; e 5. Herbicidas em V4 + pyraclostrobin + epoxiconazole $\left(66,5+25 \mathrm{~g} \mathrm{ha}^{-1}\right)$ em R2 e R5.1. Na cultivar 'Conquista' foi utilizado o graminicida: setoxydim $\left(230 \mathrm{~g} \mathrm{ha}^{-1}\right)+$ óleo mineral $(0,5 \% \mathrm{v} / \mathrm{v})$, aplicado em V3, e os latifolicidas: bentazon $\left(480 \mathrm{~g} \mathrm{ha}^{-1}\right)+$ chlorimuron-ethyl $\left(11,25 \mathrm{~g} \mathrm{ha}^{-1}\right)$, aplicados em V4. Já na cultivar 'Valiosa RR' foi utilizado 
glyphosate $\left(720 \mathrm{~g} \mathrm{ha}^{-1}\right)$, em V4. Os estádios V4, R2 e R5 correspondem a terceiro trifólio expandido, pleno florescimento e enchimento de grãos, respectivamente. A utilização de diferentes herbicidas nos tratamentos deve-se ao manejo empregado nas duas cultivares, devendo ainda ser considerada a ação indireta de herbicidas sobre a doença. A semeadura ocorreu no dia 11 de janeiro de 2006, com o intuito de propiciar maior inóculo inicial, garantindo a incidência da ferrugem asiática de forma natural. Foram realizadas adubações em quantidade equivalente a $16 \mathrm{~kg} \mathrm{ha}^{-1} \mathrm{de}$ $\mathrm{N}, 96 \mathrm{~kg} \mathrm{ha}^{-1} \mathrm{de}_{2} \mathrm{P}_{2}$ e $108 \mathrm{~kg} \mathrm{ha}^{-1} \mathrm{de} \mathrm{K}_{2} \mathrm{O}$. As aplicações foram realizadas com pulverizador pressurizado com $\mathrm{CO}_{2}$, equipado com quatro bicos duplo leque (Mícron 110DB2). A pressão utilizada foi de 40 libras pol$^{-2} \mathrm{e}$ volume de calda de $150 \mathrm{~L} \mathrm{ha}^{-1}$, a fim de se obter boa cobertura do fungicida na planta (verificado por papel hidrossensível).

Foi quantificada semanalmente a severidade de ferrugem asiática, com base na escala diagramática proposta por GODOY et al. (2006). Estimou-se a severidade por meio da média das avaliações dos terços inferior, médio e superior, em quatro plantas por parcela. $\mathrm{O}$ índice de área foliar (IAF) da soja foi determinado utilizando-se o medidor eletrônico LI-3100 (LI-COR Nebraska). Os dados obtidos por meio das avaliações da área foliar e da severidade foram utilizados para os cálculos da área foliar total por planta e da área foliar afetada pelo patógeno. A severidade em função do tempo foi analisada estatisticamente por regressões não lineares, sendo utilizado o software STATÍSTICA 6.0 (Statsoft, Tulsa). Foram testados os modelos:

$$
\begin{array}{ll}
\text { Logístico } & \mathrm{Y}=1 /\left[1+b_{1}{ }^{*} \exp \left(-b_{2}{ }^{*} \mathrm{t}\right)\right] \\
\text { Gompertz } & \mathrm{Y}=\exp \left[-b_{1}{ }^{*} \exp \left(-b_{2}{ }^{*} \mathrm{t}\right)\right] \text {, }
\end{array}
$$

em que $Y$ é a severidade da doença, $b_{1}$ é um parâmetro relacionado com o inóculo inicial [ $\mathrm{b} 1=(1 / \mathrm{yo}) / \mathrm{yo}], b_{2}$ corresponde à taxa de progresso da doença, e $t$ é o tempo, em dias após a semeadura que foram ajustados aos dados da severidade média da planta (proporção) em função do tempo (dias após a semeadura).

E Logístico com taxa variável $Y=1 /\{\{1-[1-$ $\left.\left.\left(1 / b_{1}\right)\right]^{*} \exp \left\{b_{2} / b_{3}^{*}\left[1-\exp \left(b_{3}^{*} \mathrm{t}\right)\right]\right\}\right\}$, em que $Y$ é a severidade da doença, $t$ é o tempo, em dias após a semeadura, $b_{1}, b_{2}$ e $b_{3}$ são parâmetros do modelo e foram ajustados aos dados da severidade do terço inferior da planta (proporção) em função do tempo (dias após a semeadura).

A adequação do modelo aos dados foi analisada pelos coeficientes de determinação $\left(R^{2}\right)$, obtidos pela regressão não linear e pela ausência de padrão de resíduos. Os parâmetros $b_{1}$ e $b_{2}$ obtidos de cada curva foram comparados pelo "teste t" para verificar se houve diferença significativa desses parâmetros entre os tratamentos.

A área abaixo da curva de progresso da doença (AACPD) foi calculada com base na severidade média das avaliações, enquanto que as variáveis referentes à área foliar sadia foram assim calculadas: $\mathrm{n}_{\mathrm{o}}-1$

$\left.\mathrm{HAD}=\sum_{\mathrm{i}=1}^{\sum} \operatorname{IAF}_{\mathrm{i}}\left(1-\mathrm{x}_{\mathrm{i}}\right)+\mathrm{IAF}_{i+1}\left(1-\mathrm{x}_{\mathrm{i}+1}\right)\right] / 2\left(\mathrm{t}_{\mathrm{i}+1}-\mathrm{t}_{\mathrm{i}}\right)$, em que HAD é a duração da área foliar sadia, IAF é o índice de área foliar, x é severidade, em proporção, e té o tempo em dias após os tratamentos.

$$
\begin{aligned}
& \mathrm{n}-1 \\
& \text { e HAA }=\Sigma I\left[\left(1-x_{\mathrm{i}}\right)\left(1-\exp \left(-\mathrm{kIAF}_{\mathrm{i}}\right)\right)+\left(1-\mathrm{x}_{\mathrm{i}+1}\right)(1-\exp (-\right. \\
& i=1
\end{aligned}
$$

$\left.\left.\left.\mathrm{kIAF}_{\mathrm{i}+1}\right)\right)\right] / 2\left(\mathrm{t}_{\mathrm{i}+1}-\mathrm{t}\right)$, em que HAA é absorção do tecido sadio, em dias, IA $A^{i} F$ é o índice de área foliar, $x$ é a severidade, em proporção, $k$ é o coeficiente de extinção, $I$ é a radiação global, em $\mathrm{MJ} \mathrm{m}^{-2} \mathrm{dia}^{-1}$, e t é o tempo em dias após os tratamentos. Estes foram relacionados à produção por meio de regressões lineares descritas pela equação $\mathrm{y}=a^{*} \mathrm{x}+b$, em que $\mathrm{y}$ corresponde à variável analisada, $\mathrm{x}$ é a produtividade $\left(\mathrm{kg} \mathrm{ha}^{-1}\right)$, e $a$ e $b$ são parâmetros do modelo. Os dados de radiação global (I) foram obtidos na estação meteorológica do SIMEPAR (Londrina). Foi utilizado o valor médio do período para o cálculo da equação. O valor do coeficiente de extinção (k) utilizado foi de 0,7 .

A colheita foi realizada no dia 03 de junho, sendo as sementes trilhadas e submetidas à pré-limpeza. Após esse procedimento, estas foram encaminhadas ao Laboratório de Sementes da ESALQ/USP, para determinação do teor de água e da massa de grãos produzida por parcela, para o cálculo da produtividade agrícola (teor de água de 13\%), expressa em $\mathrm{kg} \mathrm{ha}^{-1}$.

Os resultados de AACPD, HAA e HAD foram submetidos às pressuposições da ANOVA, em que foram verificados quanto à normalidade dos dados (teste de Lilliefors) e homogeneidade de variância pelo de teste de Bartlett, ambos com nível de 5\% de probabilidade. Posteriormente, foi realizada a análise de variância (ANOVA), e as médias foram comparadas pelo teste de Tukey (com nível de 5\% de probabilidade), sendo testados apenas tratamentos, uma vez que as cultivares sofreram interferência de diferentes herbicidas.

\section{RESULTADOS E DISCUSSÃO}

De acordo com os dados da área experimental, as condições climáticas foram favoráveis à ocorrência da doença. Nos meses de janeiro, fevereiro e março, a temperatura média registrada $\left(\mathrm{T}=24,5^{\circ} \mathrm{C}\right)$ foi 
adequada ao desenvolvimento da cultura e do patógeno, apresentando uma distribuição regular de chuvas. Nos meses de abril e maio, as chuvas foram mais esparsas, e a temperatura média apresentou declínio $\left(\mathrm{T}=19,8^{\circ} \mathrm{C}\right)$, influenciando o maior período de molhamento foliar e favorecendo o desenvolvimento do patógeno no campo.

Os primeiros sintomas foram observados quando a cultura estava no estádio V11, próximo ao florescimento (R1), nas duas cultivares. Os sintomas visualizados nas folhas evoluíam desde urédias isoladas a áreas coalescentes, provocando amarelecimento e abscisão foliar. De acordo com diversos autores, a ferrugem asiática pode ocorrer na fase vegetativa e reprodutiva; apresentando, entretanto, maior susceptibilidade a partir do florescimento da soja (estádio R1/R2) (YORINORI; LAZZAROTTO, 2004). Neste experimento, como a doença ocorreu em estádio anterior ao florescimento, houve severa redução na produtividade. Segundo BERGAMIN \& AMORIM (1996), a quantificação de danos também deve envolver o conhecimento do potencial de dano que uma doença pode causar. Assim, desejava-se que a incidência da ferrugem fosse precoce, o que poderia ser obtido pela semeadura fora de época e pelo aumento na pressão de inóculo do patógeno.

Os tratamentos fungicidas nos estádios R2 e R5.1 promoveram redução significativa na severidade da doença, quando comparados às parcelas não tratadas, para as duas cultivares estudadas. Ressaltase ainda que o tratamento que continha estrobilurina + triazol (pyraclostrobin + epoxiconazole $-66,5+25 \mathrm{~g} \mathrm{ha}^{-1}$ ), em R2 e R5.1, foi o que apresentou maior período residual, reduzindo a taxa de desenvolvimento da doença, quando comparado com epoxiconazole $(50 \mathrm{~g}$ $\mathrm{ha}^{-1}$ ), do grupo dos triazóis (Figura 1). Foi verificado aumento na severidade da doença durante a fase reprodutiva, o que torna relevante o posicionamento antecipado no controle, uma vez que o tratamento curativo tem baixa eficácia.

Os efeitos das pulverizações dos tratamentos foram analisados pelos modelos logístico e Gompertz para os três terços. O terço médio das plantas foi o que apresentou maior correlação com a severidade média da parcela, sendo o mais eficiente para diferenciá-los, pois a alta pressão de inóculo no terço inferior e menor severidade no terço superior fizeram com que os tratamentos não apresentassem diferenças significativas em relação às variáveis estudadas. Com relação à severidade média, o modelo logístico foi o que melhor se ajustou aos dados, visto que houve aumento na severidade em todos os tratamentos, observando-se maior taxa de progresso da doença nos tratamentos 1 e 2, ou seja, naqueles sem controle da doença (Figura 1). Ainda, verificou-se que a taxa de progresso no terço inferior foi maior que nos demais, principalmente nos tratamentos 1 e 2 (herbicidas em V4). Comparando-se o elevado coeficiente de determinação $\left(\mathrm{R}^{2}\right)$ dos modelos logístico e logístico com taxa variável, bem como os parâmetros $b_{1}$ e $b_{2}$, pelo "teste t", foi possível constatar essa diferença, sendo o modelo logístico com taxa variável mais bem ajustado aos dados (Figura 2). Com base nos resultados, pode-se afirmar que a severidade progrediu rapidamente no terço inferior, em parte pela maior dificuldade de penetração associada à condição microclimática mais propícia à ocorrência da doença, favorecendo assim a taxa de progresso da doença.

Os resultados relativos à $\mathrm{AACPD}, \mathrm{HAA}$ e HAD são apresentados na tabela 1. A partir dos resultados da AACPD, ficou evidente que tratamentos em que foram empregados fungicidas (tratamento 3, 4 e 5) foram significativamente superiores aos demais. Entre estes, destacou-se o tratamento 3 (herbicida + pyraclostrobin, em V4 e pyraclostrobin + epoxiconazole, em R2 e R5.1), que apresentou menor AACPD, devido ao maior controle exercido sobre a doença. Tal resultado pode ser atribuído à ação mais rápida e menos duradoura do triazol em relação à estrobilurina. Para a variável HAA, os tratamentos menos afetados pela doença tiveram maior capacidade de absorver a radiação solar, como foi observado novamente no tratamento 3 . Assim, salienta-se que tratamentos com fungicidas em R2 e R5.1 apresentaram melhor controle da doença, possuindo maior área foliar sadia para a absorção da radiação solar. Ainda, com relação à $\mathrm{HAD}$, os tratamentos com fungicidas em R2 e R5.1 apresentaram maior duração da área foliar sadia, em comparação com aqueles sem aplicação de fungicidas (tratamentos $1 \mathrm{e} 2$ ), indicando que a doença causou redução no período de manutenção da área foliar. Por essa razão, os tratamentos 3 (herbicida + pyraclostrobin, em V4 e pyraclostrobin + epoxiconazole, em R2 e R5.1) e 5 (herbicida em V4 e pyraclostrobin + epoxiconazole, em R2 e R5.1) diferiram novamente dos demais, bem como foi apurada diferença significativa para o tratamento 4 (herbicida + pyraclostrobin, em V4 e epoxiconazole, em R2 e R5.1), uma das razões pode ser atribuída ao maior efeito residual da estrobilurina em relação ao triazol isoladamente. Cabe ressaltar que, na cultivar 'MG/BR46 Conquista', houve diferença inclusive entre os tratamentos 1 (testemunha) e 2 (herbicida), que pode ser atribuída à ação dos herbicidas interferindo na área foliar da cultura. 


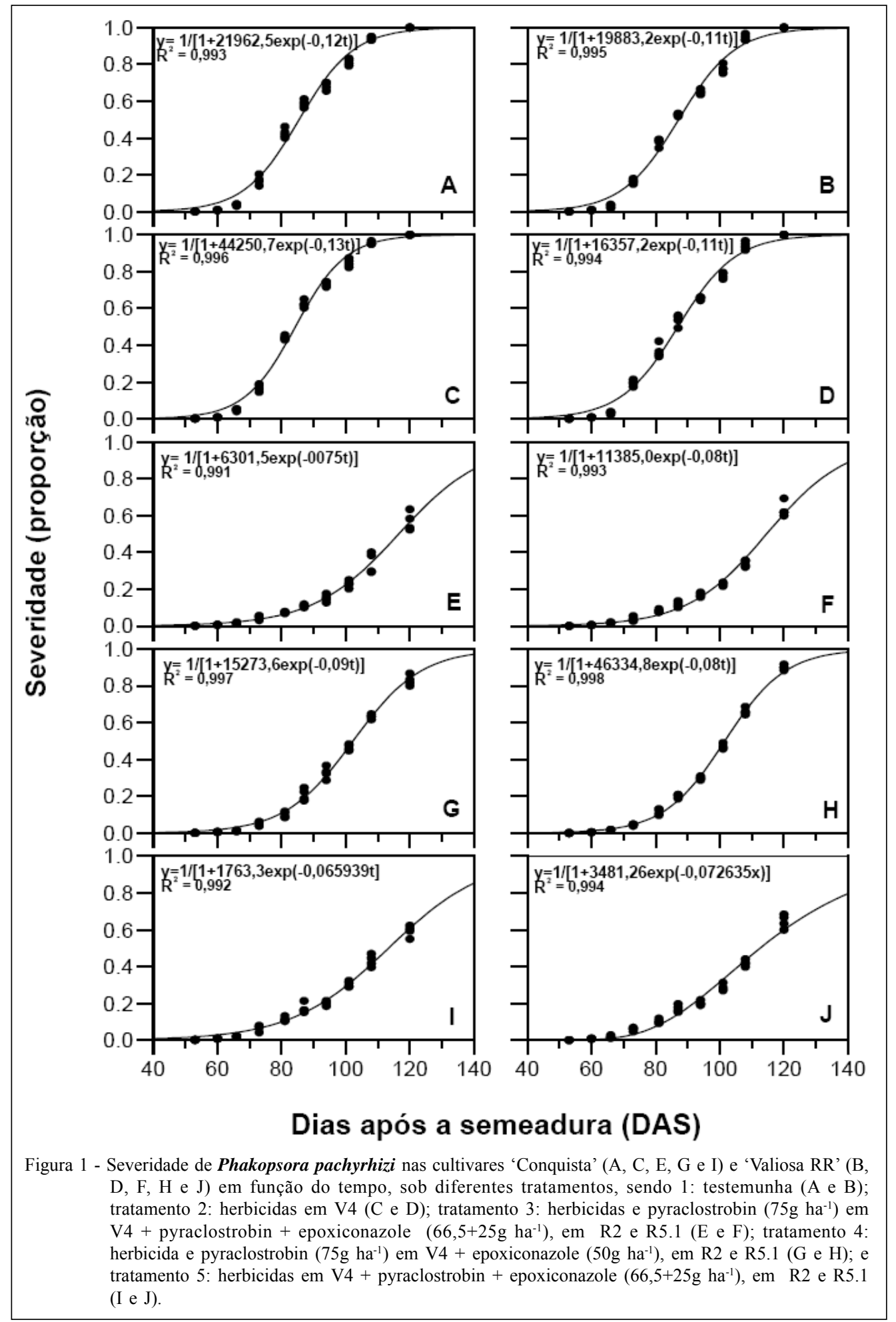

Observando-se as relações entre AACPD, HAA e HAD com a produtividade $\left(\mathrm{R}^{2}=0,98 \mathrm{em}\right.$ 'Conquista' e 0,99 em 'Valiosa RR'; 0,96 em 'Conquista' e 0,98 em 'Valiosa RR'; 0,94 em ‘Conquista' e 0,96 em
'Valiosa RR', respectivamente) pode-se inferir que a $\mathrm{AACPD}$ foi inversamente relacionada à produtividade, ao contrário de HAA e HAD. Assim, quanto maior o valor obtido pela integral da área abaixo da curva da 


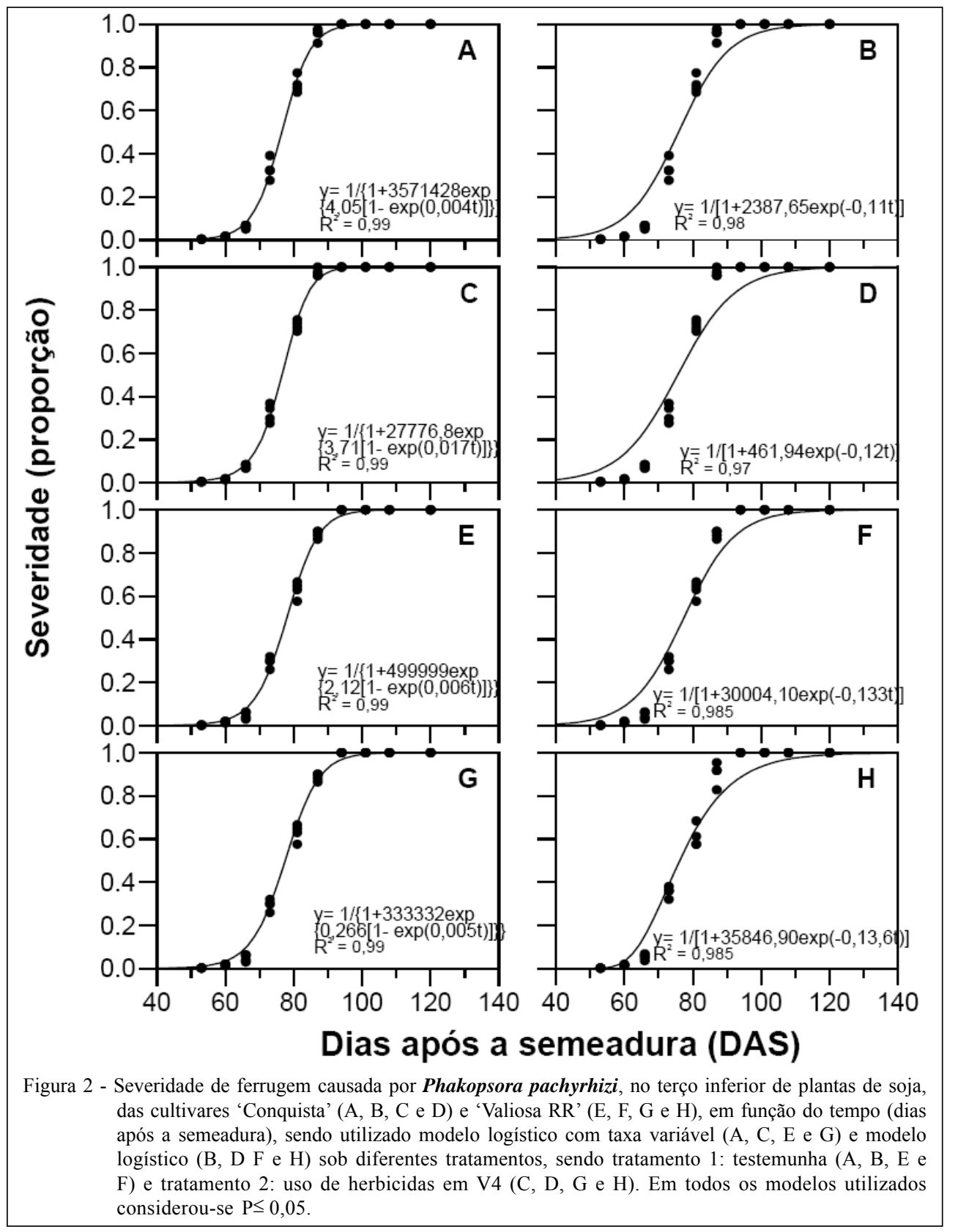

doença, menor será a produtividade nesse patossistema. Sabe-se que, em situações severas, $\boldsymbol{P}$. pachyrhizi pode afetar os componentes de produção, causando abortamento e queda de vagens e resultando na perda de produtividade da cultura da soja, como relatado por YANG et al. (1991), os quais citaram ainda que a intensidade das perdas depende da suscetibilidade da cultivar, do estádio fenológico quando a ferrugem foi estabelecida e das condições climáticas reinantes. Por essa razão, essas três variáveis estudadas podem ser consideradas mais apropriadas do que a avaliação da severidade média numa determinada fase fenológica para quantificar os danos provocados por $\boldsymbol{P}$. pachyrhizi.

\section{CONCLUSÃO}

O modelo logístico é o que mais se adapta com a severidade média das avaliações e a curva de progresso da ferrugem asiática. No terço inferior, o modelo logístico com taxa variável é o que mais se ajustou aos dados, considerando que essa posição do 
Efeito de herbicidas e fungicidas nas curvas de progresso e quantificação de danos causados pela ferrugem asiática da soja.

Tabela 1 - Área abaixo da curva de progresso da doença (AACPD), absorção do tecido sadio (HAA) e duração da área foliar sadia (HAD) nas cultivares 'Conquista' e 'Valiosa RR', sob diferentes tratamentos herbicida(s) e fungicidas'.

\begin{tabular}{|c|c|c|c|c|c|c|}
\hline \multirow[t]{2}{*}{ Tratamento } & \multicolumn{3}{|c|}{--------------------------Conquista------------------------ } & \multicolumn{3}{|c|}{-------------------------Valiosa RR----------------------' } \\
\hline & AACPD & HAA & HAD & AACPD & HAA & HAD \\
\hline 1 & $3375,78 \mathrm{a}^{2}$ & $1474,42 \mathrm{~d}$ & $169,52 \mathrm{~d}$ & $3247,29 \mathrm{a}$ & $1514,22 \mathrm{~d}$ & $171,50 \mathrm{~d}$ \\
\hline 2 & $3486,34 \mathrm{a}$ & $1410,00 \mathrm{e}$ & $153,52 \mathrm{e}$ & $3267,80 \mathrm{a}$ & $1513,32 \mathrm{~d}$ & $172,94 \mathrm{~d}$ \\
\hline 3 & $1101,69 \mathrm{~d}$ & $2065,41 \mathrm{a}$ & $253,92 \mathrm{a}$ & $1155,89 \mathrm{~d}$ & $2090,45 \mathrm{a}$ & $267,65 \mathrm{a}$ \\
\hline 4 & $1911,11 \mathrm{~b}$ & $1859,88 \mathrm{c}$ & $220,67 \mathrm{c}$ & $1966,23 \mathrm{~b}$ & $1883,71 \mathrm{c}$ & $235,49 \mathrm{c}$ \\
\hline 5 & $1378,89 \mathrm{c}$ & $1965,49 \mathrm{~b}$ & $229,98 \mathrm{~b}$ & $1372,13 \mathrm{c}$ & $2023,06 \mathrm{~b}$ & $246,69 \mathrm{~b}$ \\
\hline $\mathrm{CV}$ & 2,31 & 0,67 & 0,70 & 1,97 & 0,56 & 0,57 \\
\hline DMS & 117,15 & 26,36 & 3,23 & 97,93 & 22,74 & 2,77 \\
\hline
\end{tabular}

1 Tratamento 1: testemunha; tratamento 2: herbicidas em V4; tratamento 3: herbicidas e pyraclostrobin $\left(75 \mathrm{~g}\right.$ ha $\left.{ }^{-1}\right)$ em $\mathrm{V} 4+$ fungicida pyraclostrobin + epoxiconazole $\left(66,5+25 \mathrm{~g} \mathrm{ha}^{-1}\right)$, em R2 e R5.1; tratamento 4: herbicida e pyraclostrobin $\left(75 \mathrm{~g}\right.$ ha $\left.{ }^{-1}\right)$ em $\mathrm{V}^{2}+$ fungicida epoxiconazole $\left(50 \mathrm{~g} \mathrm{ha}^{-1}\right)$, em R2 e R5.1; e tratamento 5: herbicida em V4 + fungicida pyraclostrobin + epoxiconazole $\left(66,5+25 \mathrm{~g}\right.$ ha $\left.{ }^{-1}\right)$, em R2 e R5.1. Assaí, PR, 2006.

${ }^{2}$ Médias seguidas de mesma letra na vertical não diferem entre si pelo teste de Tukey $(\alpha=5 \%)$.

dossel favorece o desenvolvimento da doença. A pulverização da mistura pyraclostrobin + epoxiconazole (tratamento 3) é a que possibilitou maior redução na severidade de $\boldsymbol{P}$ pachyrhizi. A mistura tem melhor resultado que a aplicação isolada de triazol em $\mathrm{R} 2$ e R5.1, na AACPD, e as variáveis HAA e HAD, sendo estas consideradas apropriadas para a quantificação dos danos provocados pela ferrugem asiática. $\mathrm{Na}$ cultivar 'MG/BR-46 Conquista', a ação dos herbicidas afetaram temporariamente a área foliar, atingindo indiretamente as variáveis HAA e HAD.

\section{AGRADECIMENTOS}

A professora Lilian Amorim pelas valorosas sugestões e ao Conselho Nacional de Desenvolvimento Científico e Tecnológico (CNPq), pela bolsa de G.M. Tsumanuma.

\section{REFERÊNCIAS}

ANDERSON, J.A.; KOLMER, J.A. Rust control in glyphosate tolerant wheat following application of the herbicide glyphosate. Plant Disease, Saint Paul, v.89, p.1136-1142, 2005. Disponível em: <http://apsjournals.apsnet.org/doi/pdfplus/ 10.1094/PD-89-1136>. Acesso em 28 maio, 2010.

BERGAMIN FILHO, A. Curvas de progresso da doença. In: BERGAMIN FILHO, A. et al. (Eds.). Manual de fitopatologia: princípios e conceitos. 3.ed. São Paulo: Agronômica Ceres, 1995. V.1, cap.30, p.602-626.

CALAÇA, H.A. Ferrugem asiática da soja: relações entre o atraso do controle químico, rendimento, severidade e área foliar sadia de soja (Glycine max L. Merrill). 2007. 80f. Dissertação (Mestrado em Agronomia) - Pós graduação em Fitopatologia, Universidade de São Paulo, SP.

GODOY, C.V. et al. Diagrammatic scale for assessment of soybean rust severity. Fitopatologia brasileira, Brasília, v.31, n.1, 2006. Disponível em: <http://www.scielo.br/ scielo.php? s c ri pt = sci_art text \& pid = S $0100-$ 1582006000100011 \&lng=en\&nrm=iso>. Acesso em: 27 maio, 2010. doi: 10.1590/S0100-41582006000100011.

FENG, P.C.C. et al. Glyphosate inhibits rust diseases in glyphosate-resistant rust wheat and soybean. Proceedings of the National Academy of Science, Washington, v.102, p.17290-17295, 2005. Disponível em: <http://www.pnas.org/ content/102/48/17290.full>. Acesso em: 27 maio, 2010.

GODOY, C.V. Danos causados pela mancha angular em feijoeiro, no município de Piracicaba. $1995.72 \mathrm{f}$. Dissertação (Mestrado em Agronomia) - Pós graduação em Fitopatologia, Universidade de São Paulo, SP.

SCHMIDT, J. et al. Quantification of damages caused by the Asian soybean rust (causal agent Phakopsora pachyrhizi) based in soybean (Glycine max) physiological components. Phytopathology, Saint Paul, v.98, n.6 (Supplement), p.S141, 2008.

SINCLAIR, J.B.; HARTMAN, G.L. Soybean diseases. In: HARTMAN, G.L. et al. (Eds.). Compendium of soybean diseases. 4.ed. Saint Paul, MN: APS, 1999. p.3-4.

YANG, X.B. et al. Development of yield loss models in relation to reductions of components of soybean infected with Phakopsora pachyhizi. Phytopathology, Saint Paul, v.81, n.11, p.1420-1426, 1991.

YORINORI, J.T.; LAZZAROTTO, J.J. Situação da ferrugem asiática da soja no Brasil e na América do Sul. Londrina: Embrapa Soja, 2004. 27p. (Documentos, 236). 